\title{
LA DIALECTICA DE LA CONCIENCIA DE SÍ MISMO
}

\section{INTRODUCCIóN}

§ 1. Datos. Nada hay más cierto que el conocimiento de los propios estados de conciencia. No se puede tener certeza mayor que la certeza de que uno existe (mientras es consciente), de que uno está (ahora) viviendo tales o cuales experiencias. Por el contrario, el conocimiento del prójimo (esto es, de que existen otras personas, de que existen sus experiencias, de que éstas son para ellas plenamente ciertas, etc.), aparece, con reflexión, indirecto e incierto: tiene que fundarse, y mediante una relación oscura, en la observación de los cuerpos que nos rodean, de sus movimientos y de su situación con respecto a otros objetos, en suma, en la observación de su comportamiento y de las circunstancias en que ese comportamiento sucede.

En fin

Nadie sabe lo que una persona realmente siente -xcepto la persona misma.

Ahora bien, si lo que sabemos de otros seres pensantes y sintientes, en tanto que pensantes y sintientes, es derivativo de lo que sabemos de ciertos cuerpos físicos vecinos, de carne y hueso, ¿no es acaso posible que al final de cuentas no haya más pensamiento o sensación o sentimientos que el mío, más conciencia que la mía? ¿¿No es acaso posible que "detrás" o "debajo" de esos cuerpos humanos tan parecidos al mío, tan activos, tan habladores, no haya ni dolor, ni alegría, ni conciencia de ninguna especie? Una vez que se reconoce que uno no sabe realmente lo que el otro siente, todas estas preguntas se tornan agudas y monopolizan la atención.

¿Qué puede en efecto garantizar con plenitud las conciencias ajenas y sus experiencias? ¿Qué garantiza que los estados de conciencia ajenos, sean realmente de la misma clase o naturaleza que los estados de conciencia originarios, míos, que se dan en toda inmediatez?

¿Qué garantiza que los estados de conciencia, que yo postulo, "detrás" del comportamiento de ese cuerpo sean los mismos que la autoconciencia (por ellos constituida) aprehende originaria e inmediatamente - -según la necesidad de toda autoconciencia?

§ 2. Problema y tarea. En la vida ordinaria no puede formularse ninguna de las preguntas anteriores. Vivir, al modo cotidiano, es aceptar la existen- 
cia de los otros a través de sus acciones y sus circunstancias. "Alli" dispone uno de sus recursos, generalmente adecuados, para resolver las dudas parciales de si existe Pegaso, de si existen los tigres en África, de si existió Jesús de Nazaret, etc. En cambio las preguntas anteriores son extra-ordinarias: ellas ponen en juicio todos los recursos ordinarios para decidir si otras personas existen. Esas preguntas ponen de manifiesto que hay una laguna entre los recursos todos (acciones, circunstancias y dichos) y las conclusiones a que éstos nos llevan: existe una mente o conciencia con tales o cuales experiencias. Vivir cotidianamente es, en parte, cerrar los ojos a esa laguna y pasar sobre los puentes que la ocultan; reflexionar filosóficamente es concentrar la mirada en la laguna, acostumbrar los ojos a la oscuridad que la inunda y esforzar la vista sobre su contorno y sobre el tejido de los puentes.

La tarea filosófica consiste en "describir" esa laguna con detalle profuso y explicar por qué en la vida cotidiana siempre hay puentes que la cubren. De allí que el filósofo que se limita a gritar a voz en cuello que hay puentes no ha tomado su cargo seriamente. Este hecho, que él destaca, junto con el hecho lógico de que la laguna existe, como lo afirman el escéptico y el solipsista, constituyen el problema filosófico: explicar cómo es posible que los dos hechos co-existan, codeándose, sin estrujarse el uno al otro.

No se trata, pues, de construir un argumento irrefutable cuya conclusión sea: "Hay otros seres que piensan y sienten". Tampoco se trata de agregar información psico-sociológica que haga probable la existencia de otros seres pensantes y sintientes. Se trata, por el contrario, de entender ese conocimiento del prójimo que ya poseemos. Se trata de analizar la estructura de ese conocimiento, y ver el sentido de las preguntas extra-ordinarias acerca del prójimo que son nuestro punto de partida.

En un sentido manifiesto (cuyo análisis es cosa complicada) tener una experiencia (in latu sensu) de cierto tipo, presupone que se disponga de ciertos conceptos, los característicos del tipo de experiencia en cuestión. Así, nuestra experiencia del prójimo, de sus estados mentales y de conciencia (en el sentido en que uno puede expresarse de este modo) requiere la posesión de los conceptos mentales ordinarios. De manera que analizar nuestro conocimiento, o experiencia, del prójimo en general (en contraste con la adquisición del conocimiento de tal o cual persona) es analizar las conexiones generales entre los diversos conceptos mentales, así como las conexiones entre todos esos conceptos y aquellos que nos sirven para pensar cómo se comportan los organismos, cómo son los procesos fisiológicos en su interior, etc. Podemos decir que el problema consiste en estudiar las condiciones generales, necesarias para poder pensar en otras personas - condiciones que impregnan todo saber concreto acerca de una persona cualquiera (v. gr., nuestra madre, Napoleón I). Esas condiciones generales son precisamente las que caracterizan el conjunto de conceptos mentales como sistema de conceptos 
que hacen posible la experiencia del prójimo. Así, pues, nuestra tarea consiste en una "descripción" o análisis de la estructura de la experiencia del prójimo - independientemente de toda experiencia de una persona concreta.

\section{PRESUPOSICIONES}

§. Lenguaje. Es plausible suponer que una conciencia de sí mismo es imposible sin un lenguaje, o sistema de símbolos a través del cual se pueda organizar tal conciencia. Sin embargo, basta suponer que es posible siempre examinar los "contenidos" de una conciencia mediante el análisis de la estructura de un lenguaje en el que, en principio, esa conciencia con tales "contenidos" pudiera haberse organizado.

Así, nuestra tarea consiste en explicitar las condiciones generales necesarias para que un sistema de símbolos resulte adecuado para que una conciencia del prójimo se estructure a través de él -sea o no verdad que puede haber conciencia sin lenguaje.

$\S$ 4. Identificación. Daremos por sentado que la distinción entre individuos (o particulares) y característica (o propiedades, circunstancias, etc.), es esencial a todo lenguaje que trata de objetos materiales o sujetos de experiencia. Un lenguaje de ese tipo, diremos, presupone un universo de discurs̄o, a saber, el conjunto de los individuos especiales de que trata el lenguaje. Más específicamente, supondremos:

C) Si los individuos de un universo de discurso pueden ser: I) más de uno, 2) contables directamente (es decir, que para algún conjunto finito de ellos es empíricamente factible establecer una correspondencia entre cada individuo del conjunto y un número o numeral, entonces hay un criterio o manera de distinguir un individuo de otro).

Este principio es una modificación en dos respectos de un principio formulado por P. F. Strawson ${ }^{1}$ (Oxford).

§ 5. Dialéctica. La teoría filosófica que hay que desarrollar, diremos, describirá la dialéctica del lenguaje de lo mental. La palabra 'dialéctica' se emplea para indicar que se trata de mostrar ciertas relaciones fundamentales entre dos lenguajes o estructuras conceptuales, una montada, por así decirlo, sobre la otra.

Es corriente oír hablar de la lógica del lenguaje moral, o del lenguaje de la ciencia, o de la lógica del concepto, mente, cosa, etc. Siguiendo esa tradición, podría decirse que aquí nos proponemos desarrollar, o formular,

1 P. F. Strawson, Individuals: An Essay in Descriptive Metaphysics, Londres: Methuen \& Co. Ltd., 1959, p. 99 n, et al. 
un segmento de la lógica del lenguaje de lo mental, o de la lógica de la conciencia. Aquí por 'lógica' se quiere decir el conjunto de implicaciones que constituyen los conceptos característicos del tipo de conocimiento que nos interesa. Sin embargo, parte de lo que quiero poner de relieve es precisamente que en el caso del lenguaje de la mente no nos las habemos con un sistema de conceptos, todos en el mismo plano, vinculados por relaciones de implicación. Antes bien, nos las habemos con dos sistemas de conceptos, de modo que hay una lógica en cada sistema, pero hay además ciertas relaciones formales entre los dos sistemas, que son de otra suerte. Son esas conexiones las que me interesa discutir primordialmente. Como se trata de conexiones entre dos sistemas cada uno con su estructura lógica, creo que bien puede hablarse de las relaciones dialécticas, y, por ende, de la dialéctica de lo mental, o de la autoconciencia.

En lo dicho ya se ha anticipado algo importante. Y como se trata de una teoría, de una manera de ver el sistema de conceptos mentales, la dirección anticipada no puede ser la conclusión de un argumento. La prueba de que ese modo de ver se justifica no es un razonamiento demostrativo, sino una masa de argumentos que muestran que ciertas dificultades presentes en los "datos" que nos llevan al. problema filosófico se resuelven - sin que, por supuesto, aparezcan dificultades mayores.

§ 6. Lenguaje privado. Es necesario explicitar otra presuposición importante, ya que hay un enjambre de argumentos poderosos de Wittgenstein que pretenden probar que tal prẹsuposición es falsa. La presuposición es:

D) Es lógicamente posible que una conciencia se desarrolle aislada de toda otra conciencia.

La negación de $D$ ) es una conclusión que Wittgenstein y sus discípulos sacan de los varios argumentos del primero para probar que un lenguaje privado es imposible. En este contexto se entiende por lenguaje privado uno que se emplea para pensar objetos que sólo el hablante puede lógicamente conocer. El argumento básico de Wittgenstein puede resumirse así:

Un lenguaje es un sistema de reglas para el uso de signos. Una regla es algo que puede usarse tanto correcta como incorrectamente. .Una persona que usa un lenguaje privado tiene que usar reglas que sólo ella puede lógicamente determinar si se han empleado correctamente o no. Pero esto es imposible; por tanto, un lenguaje privado es imposible.

Lo anterior no es más que un bosquejo brevísimo. Apenas formula las premisas principales: I) que un lenguaje es un conjunto de reglas, 2) que una persona no puede por sí misma determinar que algo se ha hecho correcta 
o incorrectamente. Los varios argumentos de Wittgenstein tienen por objeto, precisamente, establecer la premisa 2).

La presentación de Wittgenstein es oscura, 2 en virtud de su estilo epigramático. Pero hay, afortunadamente, discusiones competentes de algunos de sus discípulos. ${ }^{3}$ Sin embargo, yo no estoy convencido de que los argumentos dados puedan probar la proposición 2), y tengo ciertas dudas acerca de la preposición 1). Examinaré esos argumentos detalladamente en otra parte. ${ }^{4}$

\section{DIALÉCTICA DE LA CONCIENCTA DE SÍ MISMO}

\$ 7. Preliminar: mitos filosóficos. Ya he anticipado que el lenguaje de lo mental tiene una estructura dialéctica; es un complejo de sublenguajes relacionados entre sí no meramente por implicación formal. La relación entre ellos consiste primordialmente en que varios símbolos hacen la conexión, porque representan papeles similares en cada sublenguaje. Es, para decirlo paradójicamente, una relación de equivocidad, de dualidad lógica, la que liga a los sublenguajes en una armazón compleja.

La manera más fácil de presentar esa complejidad es quizá mediante un mito filosófico (más o menos en el sentido en que Platón empleaba mitos en filosofía): es decir, mediante la descripción de la "historia" lógicamente posible de una conciencia. He puesto el vocablo 'historia' entre comillas dobles pues no quiero insinuar que realmente estemos confrontando una historia posible. Es una fantasía que permite exhibir las varias "capas" o dimensiones de los conceptos mentales ordinarios, sin sugerirse en lo más minimo que alguna persona haya jamás recorrido esas capas como estadios en su biografía. Es compatible con la descripción por venir el que, segủn alguna ley psicosociológica, física o biológica, una conciencia aislada no pueda darse, y el que, por ende, esa descripción sea imposible empíricamente. Más aún, si existe (y yo he defendido este punto de vista hasta en la aritmética ${ }^{5}$ ) lo sintético a priori, la conciencia aislada y la "historia" por contarse pueden (lógicamente) ser imposibilidades a priori -pero sintéticas. Lo im-

2 L. Wittgenstein, Philosophische Untersuchungen-Philosophical Investigations, Oxford, Blackwell Ltd., 1953; edición póstuma bilingüe con trad. al inglés de $\mathbf{E}$. $\mathbf{M}$.

3 N. Malcolm, "Discussion of Wittgenstein's Philosophical Investigations", Philosophical Review, vol. LXIII (1954): 530-559; R. Rhees, "Can There Be a Private Language?", Aristotelian Society, vol. suplementario XXVIII (1954): 77-94.

4 En "The Private Language Problem" en Paul Eduardo (ed.) The Encyclopedia of Philosophy, New York, Macmillan Co., 1967, y en "The Private Language Argument", en C. D. Rollins (ed.) Knowledge and Experience, University of Pittsburgh Press, 1963, reproducido en Bobbs-Merrill Reprint Series (Indianapolis, 1968), en R. O. Jones (ed.) The Private Language Problem, Macmillan Ltd., 1971, y en Elmer Klemke (ed.) Essays on Wittgenstein, University of Illinois Press, 1971.

5 Véase mi “' $7+5=12$ ' As a Synthetic Proposition”, Philosophy and Phenomenological Research, Vol. 21 (1960), reproducido en Robert Sleigh (ed.) Necessary Truth. Englewoods, New Jersey, Prentice-Hall, 1972. 
portante es que la "historia" es una posibilidad lógica, y eso revela mucho acerca de la estructura del conocimiento del prójimo y de la autoconciencia.

Tampoco quiero sugerir que aun cuando la "historia" por contarse fuese real, el sujeto de esa historia se daría cuenta plena de los diferentes estados. Para él podría tener siempre el carácter de una reconstrucción lógico-empírica (así como la reconstrucción de nuestras vidas antes del primer año después del nacimiento propio).

$\S 8$. Conciencia sin autoconciencia. Supongamos, pues, que hay una conciencia aislada, y supongamos de golpe que esa conciencia, que habrá de llamarse conciencia de aquí en adelante, conoce de objetos y procesos físicos así como de sus propias experiencias o estados. Diremos que se encuentra en la fase 0, en que ninguna tiene conciencia de sí misma. Los dolores y las sensaciones de todas clases son para ella todos "objetos" que pertenecen a su mundo, al mundo, pues $i$ ) no hay para ella ningún otro mundo posible, y ii) ella no es parte de ese mundo: ella no sabe nada de sí misma. La conciencia es simplemente la unidad del mundo y la conciencia de esa unidad del mundo, es el yo trascendental de Kant.

La conciencia distinguirá los objetos que constituyen el mundo por sus diferentes cualidades y relaciones. Podemos imaginarla en poder de un gran sistema clasificativo en que se separan los objetos y procesos físicos de los objetos o procesos mentales. Mas lo característico de esa conciencia es que, tanto los procesos y objetos físicos como los objetos y procesos mentales (aun los que pueden considerarse como trocitos de conciencia), son (para ella) impersonales. Esa conciencia es fundamentalmente contemplativa, y hasta sus propias acciones o voliciones aparecen impersonalmente. Las proposiciones a través de las cuales se organiza, esto es, las proposiciones constitutivas del lenguaje sobre el cual se estructura, son principalmente como las siguientes, más las combinaciones de ellas mediante los signos lógicos:

a) "Hay una casa"; "Hay un dolor"; "Hay una sensación de rojo"; "Hay un sentimiento de tristeza"; "Hay un recuerdo de una mesa roja"; "Hay una reflexión sobre una casa", etc.

b) "La casa es roja"; "El dolor es agudo"; "La tristeza es persistente"; "El recuerdo de la casa es vivo", etc.

c) "La casa está junto al río"; "El dolor está en la pierna gorda"; "La sensación de rojo está relacionada con este ojo"; "La sensación de suavidad está en esa mano"; "La reflexión sobre la casa no está en ninguna parte", etc.

En general, lo característico de esa fase es que en el lenguaje de esa conciencia no pueden figurar símbolos como 'yo', 'tú', ni los conceptos de conciencia, mente, persona, etc. 
Ante ella habrá relaciones causales, de necesidad de sucesión, en los cambios de los objetos mentales, y habrá relaciones causales entre los dos. Eso le permitirá anticipar (predecir) cambios. Dados ciertos objetos y sus condiciones tendrá que haber ciertos (otros) objetos y tales o cuales condiciones. Pero habrá relaciones muy peculiares: serán como de necesidad, pero serán falibles, y la conciencia sólo oscuramente podrá distinguirlas entre sí. Esa es la forma en que se le aparecerán los propósitos y las acciones propias. Por ejemplo, su mundo contendrá un fragmento que se formulará así:

"Hay un dolor en ese brazo; debe desaparecer; si el brazo se mete en esa cubeta con... y el cuerpo a que pertenece traga..., ese dolor desaparecerá; el brazo debe meterse en la cubeta y el cuerpo debe tragar...; hay una sensación que a menudo acompaña el brazo cuando se mueve sin que nada lo empuje, etc."

De nuevo, no hay una conexión íntima entre los movimientos del brazo, las sensaciones que acompañan esos movimientos, o la presencia del dolor. Todo es impersonal. La única unidad que existe es la gran unidad de la experiencia y la unidad de cada individuo diferenciado, sea mental, sea físico; pero ni los objetos y procesos físicos forman una unidad, ni los objetos y procesos mentales forman otra, a pesar de que la diferencia pueda habérsele dado ya a la conciencia. Pero se trata simplemente de dos órdenes de individuos, que se rigen por el principio meta-lógico $(C)$.

Por supuesto, la conciencia en cierto sentido es consciente de sí misma, a saber: en el sentido de que cada una de sus experiencias u operaciones puede ser pensada por ella. Pero no puede pensarlas como suyas. Cada sensación, cada estado mental, cada reflexión de que ella es consciente es, por este mismo hecho, un objeto más en el mundo. Pero ella no puede saber nada de ese proceso de creación. Ella no está en el mundo, simplemente no existe (para sí), de modo que cada objeto es dado, sea mental, sea físico. (Y le es dado completamente, aun cuando ella sólo operase mediante un conjunto especial de categorías, según creía Kant; pues esas categorías no existen para ella como categorías para que sea consciente de objetos. Si filosofase, esas categorías serían simplemente aspectos del mundo.)

$\S$ 9. Conciencia con un yo sin conciencia de si. La conciencia pasa a la fase $r$. Desarrolla, primero, un concepto nuevo: el de un objeto especial, que viene con toda una familia de predicados correspondientes. Ese individuo es el yo, y los predicados son obtenidos de los predicados mentales, es decir, de los que se aplican a los individuos mentales. El lenguaje de la conciencia se enriquece mediante la definición de ' $y o$ ' y una lista infinita de esquemas de traducción ilustrados en la definición 2: 
Def. 1. Yo - la totalidad de los individuos mentales.

Def. 2. Yo siente un dolor $=$ hay un dolor.

Yo siente un dolor $=$ hay un dolor en esa pierna $=$ esa pierna. $Y o$ ve rojo = Hay una sensación de rojo.

Yo recuerda que $=$ Hay un recuerdo de que la casa roja era la casa roja era de dos pisos de dos pisos.

Etcétera.

En rigor, son varios los modos en que la conciencia puede enriquecer su mundo conforme al método ilustrado en Def. 1 y Def. 2. Puede introducir el concepto de un yo sensitivo, de un yo percipiente, etc., tomando la totalidad de una clase especial de objetos mentales. Lo característico de esas definiciones y reglas de traducción consiste en que cada extensión del lenguaje original es un sublenguaje trivial, porque cada una de sus proposiciones (o juicios) es traducible sin pérdida de significado en una proposición (o juicio) del lenguaje original de la fase 0 . Como Def. 1 muestra, el sublenguaje adicional contiene un universo de discurso que está lógicamente limitado a un miembro; por tanto, para los nuevos predicados mentales no vale el principio (C) formulado. ' $Y o^{\prime}$ ' es simple y llanamente el nombre propio de ese objeto único.

La conciencia todavía en esta fase introduce otro nombre propio:

Def. 3. Cuerpo = La totalidad de objetos físicos en que se presenta algún individuo mental, es decir, alguna "parte" de yo.

Una vez.más, otros "cuerpos" podrían ser introducidos según los diferentes Yoes, o clases de individuos mentales, que se prefiera considerar. $\mathbf{Y}$ es una cuestión empírica si todos esos individuos físicos llamados "cuerpos" son el mismo o no. De todos modos, lo importante es otra cosa. La Def. I hace del Yo una entidad gratuita, pues la totalidad de individuos mentales siempre existe, y realmente no se agrega nada dándole un nombre especial. En cambio, la. Def. 3 no hace del cuerpo una entidad gratuita. En primer término, es una cuestión empírica el que haya alguna relación entre individuos mentales e individuos físicos (dentro del lenguaje o conciencia de las fases 0 y I). En segundo término, el cuerpo no es. (necesariamente) la totalidad de lo físico, de modo que la conciencia no tiene otra manera de separar ese objeto material de los otros: mediante las posiciones relativas' en tiempo y espacio de las partes del cuerpo. Las proposiciones acerca del cuerpo no son eliminables. La Def. 3 no introduce un sublenguaje especial, sino meramente un vocablo que sirve dè abreviatura. La Def. 2 es una secuencia infinita de definiciones en contexto, que efectivamente enriquecen la sintaxis del lenguaje original. 
Finalmente, la conciencia introduce el sustantivo 'cuerpo'. Puede introducirlo de dos maneras distintas: a) definido como abreviatura de objeto material con características...', donde los puntos suspensivos representan una lista de predicados puramente físicos; $b$ ) definido como sigue:

Def. 4. $x$ es un cuerpo $=x$ es un objeto material que se parece al cuerpo en...

La diferencia es de poca monta. Los cuerpos, en cualquier forma que se les conciba, no son entidades gratuitas. Son objetos perfectamente delineados dentro de la experiencia de la conciencia en la fase 0 ; la diferencia está en que ahora la conciencia los piensa bajo un símbolo especial. En otras palabras, el úniverso de discurso de la fase 0 es el mismo del lenguaje de los cuerpos (dejando de lado al Yo, eliminable).

Así, pues, en su paso a la fase 1 , la conciencia no enriquece su haber empírico. Enriquece su manera de concebir los individuos mentales, y hasta puede modificar un poco su manera de concebir los objetos materiales. Los individuos mentales devienen "partes", "átomos" de un individuo especial; los objetos materiales entran bajo la luz de una relación con ese superindividuo mental: asociados con él (el cuerpo y sus partes), semejantes de varios modos a los así asociados, y del todo diferentes. Pero allí concluye el asunto. La conciencia ha ganado, creado, una perspectiva nueva del mundo, y de seguro aparecen nuevos individuos mentales (por ejemplo, nuevas sensaciones y actitudes y sentimientos de expectativa) que se originan del mero uso de un nuevo sistema conceptual, por vacio que éste sea. Pero la conciencia sigue ignorante de sí misma, de sus creaciones y sus propios estados y experiencias; aun las que dependen de su nueva estructura formal, sólo pueden presentársele individualizadas, como partes del mundo, como partes que por simple yuxtaposición constituyen el $Y o$. No puede relacionar esas sensaciones y expectativas nuevas a su nueva estructura, y, aunque sienta una diferencia sutil, inexplicable, la nueva estructura y la vieja son equivalentes: la una es siempre reducible a la otra.

En la fase I hay, pues, dos dimensiones importantes de solipsismo: a) el solipsismo inefable, inconcebible de la conciencia, y b) el solipsismo lógico necesario del Yo. En cierto sentido no se trata más que de un mismo solipsismo; pero en la fase 1, la conciencia lo sabe todo, excepto cuanto se refiera a ella como conciencia; todo lo que ella sabe de si lo sabe como cosas del Yo; pero no tiene la menor sospecha de la relación entre el $Y o$ y ella. Por el otro lado, el $Y o$ no sabe nada, sino en un sentido derivativo y vacio: el requerido por la Def. 2. Al Yo le sucede el saber, como le sucede todo lo mental, incluso las decisiones.

La relación entre el Yo y el cuerpo, o los cuerpos, sigue siendo pura- 
mente empírica. Nada se ha alterado en cuanto a las relaciones entre individuos de los dos órdenes de la fase 0 . Las decisiones y propósitos siguen siendo casos especiales en que la posesión por parte de ciertos individuos mentales de una característica peculiar de necesidad, requiere lógicamente que ciertos sucesos del cuerpo posean también esa característica; y a menudo tales sucesos se realizan, y el individuo mental se desvanece.

$\S 10$. Conciencia de si mismo. La conciencia sigue "su marcha": llega a la fase 2. Aquí irrumpe una gran revelación, cuyos orígenes se encuentran bastante "atrás". Ya desde la fase 0, la conciencia había estado acumulando un saber creciente del mundo. En efecto, ese saber está en el fondo de las definiciones 3 y 4 . Las múltiples asociaciones entre los individuos mentales y los individuos materiales o físicos habían estado en el centro de su atención desde un principio. $Y$ es de suponer que había probado diferentes hipótesis con el resultado de que muchísimas generalizaciones empíricas se encuentran ya en su posesión aunque posiblemente la mayoría de ellas no aparezca en el mundo en reflexiones individualizadas, sino que se manifiestan como modos de inferencia, como modos en que un pensamiento anticipa a otro. Pero en los últimos "momentos" de la fase 1 , los cuerpos, individualizados, aparecen en el centro de cambios notables, semejantes a los cambios del cuerpo relacionados estrechamente con individuos mentales. $Y$. la conciencia hace algo completamente distinto. No se limita a experimentar con generalizaciones hipotéticas acerca de las condiciones de los individuos, mentales o físicos, con que hasta ahora ha tratado. En su afán por "explicar" esos cambios, por producir un orden general en el mundo, crea una teoría cientifica, esto es, un lenguaje con contenido empírico irreductible al lenguaje de la fase previa.

Al lenguaje presente se agrega, de manera especial, un sistema de conceptos que se aplica a un universo de discurso completamente nuevo, cuyos individuos no son de ningún modo reducibles a los individuos previos. Además, hay reląciones empíricas entronizadas en el sublenguaje nuevo, que permiten el descubrimiento de nuevas relaciones tanto entre las nuevas entidades como entre ellas y los antiguos individuos. Esto último es lo que justifica, al final de cuentas, el empleo de la nueva estructura conceptual. Veamos su lineamiento general.

La teoría consiste esencialmente en la postulación de seres semejantes al Yo, asociados con los otros cuerpos de manera análoga a la cual el Yo y el cuerpo están asociados. Claro está, puede también incluir la postulación de individuos mentales básicos, como los que constituyen al Yo (sensaciones, dolores, etc.), siempre en relación con objetos físicos semejantes, más lejanamente, al cuerpo, pero aislados, sin constituir un individuo complejo como el Yo. Un ejemplo se da en los dolores o sensaciones desagradables atribui- 
das a un insecto o gusano, que no implican la adjudicación de una mente o un yo al insecto o al gusano. Pero el concepto fundamental, el que constituye la nueva categoría de individuos en el yo, mente, conciencia, persona, espíritu (o como quiera llamársele). Este concepto, digamos, el de $Y o$, se relaciona con el nombre propio ' $Y o$ ' de manera análoga a como el concepto de cuerpo se relaciona con el nombre propio 'cuerpo'. Pero aquí se acaba la semejanza; pues en el mundo de la fase i estaban ya todos los cuerpos que se quisieran, mientras que los otros yoes tendrán que ser creados de la nada (por así decirlo), y eso es, precisamente, la revolución de la conciencia en su paso a la fase 2. Ahora bien, la "creación" de todos esos individuos nuevos requiere la introducción de características o condiciones que los constituyan. Como son análogos al viejo $Y o$, el lenguaje nuevo de la conciencia tiene que introducir predicados análogos a los viejos predicados, esto es, se cambia el significado de los predicados mentales de modo que ya no se apliquen sólo a un individuo, cuya unicidad es una necesidad lógica, sino que se puedan aplicar a muchos otros individuos. $Y$ ese cambio de significado es verdaderamente notable; pues en la fase 1 era sencillamente una contradicción en términos decir que había un individuo mental distinto del Yo; o, más específicamente, era una contradicción en términos el que la conciencia pensara que había un dolor, un disgusto o una alegría, que no formaran parte del Yo. En cambio en la fase 2 esto se convierte en el nuevo significado de los términos 'dolor', 'alegría', 'disgusto', etc., una cuestión puramente empírica que puede ser incluso verdad gran número de veces.

Así, pues, hay que distinguir entre:

(a) Yo tiene un dolor en la pierna [fase 1]; y

(b) Yo tiene un dolor en la pierna [fase 2].

Podriamos indicar, tentativamente, la diferencia escribiendo:

(a) Yo tiene un dolor en la pierna;

(b) Yo tiene un dolor de la pierna.

Pero con la introducción de otros yoes, la vieja definición Def. I que intro dujo el vocablo ' $Y o^{\prime}$ en la fase 1 se hace arcaica. El $Y o$ no puede ser ya la totalidad de los objetos mentales. El Yo se empobrece, mas con ello se gana el derecho a ser un ente efectivo. En la fase 2 el Yo gratuito, eliminable mediante la Def. 1, desaparece, y en su lugar se alza el yo de la conciencia (que no es otra cosa, en el fondo, que la conciencia misma como entidad real). Por tanto, si continúa el signo ' $Y O^{\prime}$ ' en uso, su significado tiene que ser distinto en las proposiciones (a) y $(b)$-a pesar de que en cierto sentido en los dos casos sigue siendo el nombre del mismo ser.

Ahora bien, la proposición (a), pero no la $(b)$, es equivalente a la proposición: 
(a. 1) Hay un dolor en la pierna [fase I].

Pero (a.1) también cambia de significado en la fase 2. La conciencia ya no puede pensar en dolores, tristezas, alegrías, recuerdos, etc., a secas; tiene que pensarlo en relación con uno u otro de los yoes posibles (o reales). Por tanto, todas las formas impersonales de lo mental desaparecen en la fase 2. Los individuos mentales originarios acaban perdiendo su independencia, hasta su individualidad, para quedar supeditados a los nuevos individuos mentales. Son los yoes (o mentes, espíritus) los que vienen a compararse con las cosas materiales. La revolución en el mundo de la conciencia ha sido, pues, radical.

Con la eliminación de los hechos mentales originarios impersonales, la conciencia y el $Y_{O}$ vienen a identificarse. Los únicos hechos mentales que la conciencia puede pensar originariamente, los que en el fondo la constituyen, son los que pensaba en el estilo simple de la fase 0 o en cualquiera de los dos estilos posibles en la fase .1; pero todos ellos constituyen el yo, y sólo pueden, en la fase 2, pensarse como pertenecientes al Yo. Por ello, además, las proposiciones sobre el Yo típicas de la fase 2, como (b) arriba, tienen una dualidad peculiar. De una parte, son otros tantos casos de proposiciones que adscriben estados de conciencia o experiencias a un sujeto; de otra parte, son proposiciones mentales de las fases 0 y 1. En cambio las proposiciones que adscriben estados mentales o de conciencia a otros yoes (o personas) se mantienen "limpias" de toda contaminación con formas caducas de conciencia.

A cada proposición como (b) corresponde una proposición como (a); mientras que a una proposición sobre otro yo, análoga a $(b)$, no corresponde ninguna proposición análoga a (a). He ahí la dualidad lógica, o estructura dialéctica del lenguaje de lo mental. De un lado está el sublenguaje de las proposiciones como (b), juntamente con las que tratan de otros yoes; del otro lado está el sublenguaje de las proposiciones como (a), y entre ambos las relaciones de equivocidad a través de los predicados mentales y del signo ' $Y o$ '. El primero constituye la dimensión solipsista "primigenia", lógica, ya que no psicológicamente; el segundo es la dimensión multisubjetiva o intersubjetiva, en que la conciencia realmente se conoce, conociéndose como un yo, rodeado (posiblemente) por muchos otros yoes.

$\S 11$. Conciencia del tu: comunicación. Las transformaciones radicales de la conciencia, del $Y o$, del mundo y del lenguaje, que hemos visto no son toda la "historia". La postulación de otros yoes es precisamente la postulación de otros seres como el Yo, especialmente como el Yo en que piensan, sienten, en que hacen uso del lenguaje para pensar en sí mismos. Son seres con los que se puede hablar. Esto implica que el lenguaje que estructura la conciencia se enriquece con una nueva función: puede servir para hablar-con 
alguien, a alguien, ya no meramente para hablar o pensar de los objetos mentales o físicos. Se requiere, pues, algún signo, o mecanismo especial, para hacer la diferencia entre las dos funciones del lenguaje. Esa distinción es precisamente la que simboliza la segunda persona gramatical, ya sea en la forma de pronombres, ya sea en la forma de inflexiones verbales, ya sea mediante la entonación y pausa del vocativo, etc. El mecanismo gramatical específico carece de importancia; lo que importa es que el lenguaje en la fase 2 incluye un mecanismo cualquiera para hacer la distinción. Y ésa es, en efecto, una de las piezas fundamentales del lenguaje de la teoría científica de la existencia del prójimo, pues allí se encuentran las mejores posibilidades empíricas de verificación -indispensables a toda teoría científica.

El nuevo sistema de conceptos requiere que algunas generalizaciones entre los sucesos del cuerpo y los del Yo se apliquen a los demás yoes y a sus cuerpos. $Y$ se pueden verificar no sólo mediante la observación de los cuerpos, sino, y muy especialmente, hablando con los otros yoes y preguntándoles acerca de sus experiencias o estados mentales. Las probabilidades de error varian, al igual que en toda investigación científica, pero lo importante es que hay medios empíricos de verificación. [Desgraciadamente no puedo entrar en mayores detalles sobre este tópico en esta ocasión.]

Ahora bien, vale la peria notar que la nueva función del lenguaje puede producir, y ha producido de hecho en las lenguas ordinarias, una nueva complejidad en el lenguaje mental. La complejidad de la segunda persona gramatical es mental. La complejidad de la segunda persona gramatical es lógicamente necesaria. La complejidad de la primera persona gramatical no es estrictamente necesaria, pero sí es la más práctica. Para que haya comunicación entre dos yoes (mentes o almas) acerca de ellas mismas es necesario: i) que cada yo use un nombre propio, o símbolo especial, para pensar en sí mismo, y ii) que los demás, la conciencia de que hemos venido hablando, por ejemplo, estén en condición de saber qué nombre se da a cada yo con el cual entra en comunicación. Los mejores nombres de los yoes son aquellos que les permiten a los otros yoes saber de antemano que son nombres de tales yoes. Mas es imposible que cada yo, o la conciencia, por ejemplo, disponga de los nombres propios que los yoes se dan a sí mismos, excepto que tales nombres propios satisfagan algún principio o regla, que ya los yoes todos poseen - siempre que el lenguaje sea común, por supuesto. Los nombres más prácticos son. Dues, aguellos que se resumen en alguna fórmula. 
sí mismos, y que ese signo haga alusión a los proto-yoes de la fase 1. Así, el viejo signo ' $Y o$ ' con su doble significado ya examinado viene finalmente a desaparecer en favor de un signo todavía más complejo: a) que por un lado es el nombre propio del Yo de la fase 2, pero b) que por otro lado es un término común, al alcance de cada uno de los otros yoes como nombre propio de sí mismo. Ese signo peculiarísimo es (en español) el vocablo 'yo'.

El término 'yo' y sus compañeros 'me', 'mi', 'conmigo', no son pronombres en exactamente el mismo sentido en que todos los otros pronombres lo son, aun los personales 'tú', 'él' y los compañeros respectivos. Éstos pueden referirse en cada ocasión de su uso, a un individuo distinto; en cam. bio, 'yo', 'me' y 'mí tienen que referirse al mismo individuo siempre que una persona dada, o un yo determinado, los usa. Para cada uno de nosotros el vocablo 'yo' es sencillamente nuestro nombre propio; es el nombre propio fundamental en términos, por cuanto a través de él hacemos que los demás aprendan a llamarnos de muchos otros modos. Nos presentamos diciendo "(Yo) soy Carlos..."

En consecuencia, una proposición como "(Yo) tengo un dolor en la pierna" tiene un significado tridimensional; (a) pertenece al sublenguaje solipsista, en que es reformulable (para el caso de nuestra conciencia, según el mito anterior) como "Yo tiene un dolor en la pierna"; $(b)$ pertenece al lenguaje multisubjetivo (individual), en que (de nuevo según los términos de nuestro mito) puede reformularse como "Yo tengo un dolor en la pierna"; y (c) pertenece al lenguaje intersubjetivo, esto es, el multisubjetivo en función comunicativa, por cuanto contiene el nombre propio común.

$\S$ 12. Cuerpo y conciencia. Lo esencial en el sublenguaje multisubjetivo consiste en que los nuevos individuos, es decir, las mentes o yoes; han de relacionarse con ciertos cuerpos de la misma manera general en que el $Y o$ se relaciona con el cuerpo. Así, pues, la relación entre cada yo y su cuerpo es empírica. Sin embargo, no puede ser meramente empírica. La nueva estructura conceptual introduce un universo de discurso que puede en principio incluir cuantos individuos se quiera; pueden darse en conjuntos contables directamente. Vale para los yoes el principio $(C)$. Tiene, pues, que haber procedimientos adecuados para distinguir unos yoes de otros. Como todos los individuos mentales originarios (aquellos que pueden pensarse con el lenguaje de la fase 0 ) son partes del Yo, la única vía de la conciencia para llegar a los nuevos individuos paralelos al Yo consiste en usar los cuerpos correspondientes. Por tanto, es lógicamente necesario que la conciencia identifique otros yoes a través del comportamiento de los cuerpos correspondientes a tales yoes.

A mayor complejidad, la analogía fundamental en el lenguaje de la 
teoría de los yoes requiere que sea posible que otros yoes se relacionen con los yoes. Por tanto, es posible que algunos yoes conozcan del Yo, y esto requiere que haya también una relación lógica entre el Yo y el cuerpo, análoga a la explicada entre los otros yoes y los otros cuerpos.

En resumen, la relación entre cuerpo y mente es una trama de nexos lógicos y de asociaciones empíricas. Cada concepto mental (por ejemplo, el de dolor) se relaciona empíricamente con los conceptos físicos; cada proposición mental dada (sintética, por supuesto) se relaciona empíricamente con las proposiciones físicas. De una proposición que adscribe un dolor a alguien no se puede deducir nada acerca de las condiciones efectivas del cuerpo correspondiente: ni al revés. Sin embargo, la relación entre el sistema total de conceptos mentales y el sistema de conceptos aplicables a los cuerpos es lógica. Siempre existe la posibilidad de que una persona no sufra ningún dolor, cualesquiera que sean sus circunstancias y conducta. Sin embargo, la descripción de su dolor o de la ausencia de éste, y la descripción de la situación de su cuerpo presuponen, esto es, sólo son posibles en su inconexión porque hay un fondo de conexiones a priori que ligan los dos sistemas de conceptos:

A) Una relación a priori gnoseológica entre la mente o yo y el cuerpo -nada se puede saber de una mente excepto a través de un cuerpo;

$B$ ) Una relación a priori teorética (o epistemológica) entre lo mental ajeno y lo corporal - lo mental explica lo que pasa entre lo corporal;

c) Una relación a priori pragmática - la "aceptación" o introducción de un lenguaje o estructura conceptual consiste en la aceptación de la existencia de objetos del tipo correspondiente al lenguaje: en el caso normal no se pone en duda la presencia de una mente y sus experiencias "detrás" de un cuerpo activo;

D) Una relación a priori sistemática -en la mayoría de los casos la evidencia de un cuerpo es suficiente para establecer la presencia de una mente; esto es, ciertas proposiciones que conectan los dos sistemas son lógicamente verdaderas o analíticas; en nuestro caso tienen, especialmente, las formas siguientes:

"Si un yo vive una experiencia del tipo E, es probable en alto grado que el cuerpo correspondiente esté en situación S";

"Si un cuerpo relacionado con un yo se encuentra en situación S, entonces es altamente probable que el yo esté con experiencia E";

"Si el objeto físico $\mathrm{X}$ ha parecido conversar sobre temas diversos durante un periodo $\mathrm{T}$ y ha empleado su lenguaje aparente en actividades variadas de la manera en que una persona (o un cuerpo con un yo) lo hace, entonces es probable que $\mathrm{X}$ sea el cuerpo de un yo (o mente), o que $\mathrm{X}$ sea una persona".

E) Relación a priori ontológica entre la autoconciencia y la conciencia 
del prójimo - la verdadera autoconciencia es la conciencia de sí mismo que se desarrolla en la fase 2, esto es, cuando la conciencia es la conciencia de hechos de la forma "Yo tengo (soy, etc.)..." en que el Yo pensado no es una entidad gratuita; la autoconciencia plena presupone la posibilidad de un mundo de cuerpos u objetos materiales; más aún, presupone el haber estado asociada con algún cuerpo;

F) Presuposiciones ontológicas: Las proposiciones "Hay mentes (o yoes)" y "Hay cuerpos" son a priori en el lenguaje ordinario de lo mental; su función es análoga a la de la fórmula " $(x)(f x v-f x)$ " en las formulaciones corrientes de la lógica de predicados; "Hay mentes" incluye el término 'mente' en el sentido que se le puede dar en la fase 2, y no implica que hay otra mente, además del $Y o$; es una proposición sintética, por cuanto que el lenguaje de lo mental: es una teoría científica y como tal admite alternativas.

En ninguno de esos elementos a priori queda implícito que el concepto de persona sea más básico que el de conciencia o yo. En la presente concepción de la estructura de lo mental es perfectamente posible que una conciencia se dé descarnada. Uná conciencia tal no podría llegar a la etapa multisubjetiva de la fase 2. Por supuesto, también es posible que no se produzca ninguna conciencia descarnada. Lo importante es darse cuenta de que éstas son cuestiones empíricas.

Héctor-Neri Castañeda

INDIANA: UNIVERSITY

U.S.A. 